OPEN ACCESS

Edited by:

Gang Liu,

Xiamen University, China

Reviewed by:

Paolo Bigini,

Mario Negri Institute

for Pharmacological Research

(IRCCS), Italy

Clara Mattu,

Politecnico di Torino, Italy

${ }^{*}$ Correspondence:

Ruxandra Gref

ruxandra.gref@universite-paris-

saclay.fr;

ruxandra.gref@u-psud.fr

Specialty section:

This article was submitted to

Nanobiotechnology,

a section of the journal

Frontiers in Bioengineering and

Biotechnology

Received: 28 May 2020

Accepted: 06 August 2020

Published: 15 September 2020

Citation:

Li X, Salzano G, Qiu J, Menard M,

Berg K, Theodossiou T, Ladavière $C$

and Gref R (2020) Drug-Loaded

Lipid-Coated Hybrid

Organic-Inorganic "Stealth"

Nanoparticles for Cancer Therapy.

Front. Bioeng. Biotechnol. 8:1027.

doi: 10.3389/fbioe.2020.01027

\section{Drug-Loaded Lipid-Coated Hybrid Organic-Inorganic "Stealth" Nanoparticles for Cancer Therapy}

\author{
Xue Li', Giuseppina Salzano', Jingwen Qiu', Mathilde Menard'2, Kristian Berg'2, \\ Theodossis Theodossiou ${ }^{2}$, Catherine Ladavière ${ }^{3}$ and Ruxandra Gref ${ }^{1 *}$ \\ 1 Université Paris-Saclay, CNRS UMR 8214, Institut des Sciences Moléculaires d'Orsay, Orsay, France, ${ }^{2}$ Department \\ of Radiation Biology, Institute for Cancer Research, Oslo University Hospital, Oslo, Norway, ${ }^{3}$ University of Lyon, CNRS, UMR \\ 5223, IMP, Villeurbanne, France
}

Hybrid porous nanoscale metal organic frameworks (nanoMOFs) made of iron trimesate are attracting increasing interest as drug carriers, due to their high drug loading capacity, biodegradability, and biocompatibility. NanoMOF surface modification to prevent clearance by the innate immune system remains still challenging in reason of their high porosity and biodegradable character. Herein, FDA-approved lipids and poly(ethylene glycol) (PEG)-lipid conjugates were used to engineer the surface of nanoMOFs by a rapid and convenient solvent-exchange deposition method. The resulting lipid-coated nanoMOFs were extensively characterized. For the first time, we show that nanoMOF surface modification with lipids affords a better control over drug release and their degradation in biological media. Moreover, when loaded with the anticancer drug Gem-MP (Gemcitabine-monophosphate), iron trimesate nanoMOFs acted as "Trojan horses" carrying the drug inside cancer cells to eradicate them. Most interestingly, the PEG-coated nanoMOFs escaped the capture by macrophages. In a nutshell, versatile PEG-based lipid shells control cell interactions and open perspectives for drug targeting.

Keywords: metal organic frameworks, nanoparticles, lipids, poly(ethylene glycol), stealth, sustained drug release

\section{INTRODUCTION}

Despite progresses in drug development and cancer biology, cancer mortality rate remains over $30 \%$, and the morbidity much higher. Nanomedicine has shown great promise through drug delivery by achieving drug transcytosis, drug targeting and theranostics (Rosenblum et al., 2018; Senapati et al., 2018). Nanoscale metal organic frameworks (nanoMOFs) recently emerged as an attracting class of hybrid nanomaterials for biomedical applications due to their biodegradability, biocompatibility, elevated drug loading capacity and high versatility in terms of architecture and physico-chemical properties (Horcajada et al., 2010, 2012; He et al., 2015; Rojas et al., 2019). NanoMOFs are formed by the self-assembly of metal centers and organic ligands, leading to the formation of open crystalline structures with regular and high porosities. 
Iron (III) trimesate nanoMOFs (Figure 1 upper panel) are among the most widely studied MOFs for drug delivery (Horcajada et al., 2010; Agostoni et al., 2013; Baati et al., 2013; SimonYarza et al., 2016; Li et al., 2019a). Recently, they were shown to display several intrinsic properties of main interest in the nanomedicine field: radio-enhancement properties when submitted to $\gamma$-irradiation (Li et al., 2019a); they behaved as $\mathrm{T}_{2}$ weighted MRI imaging contrast agents (Horcajada et al., 2010) and they had intrinsic antibacterial effects killing intracellular bacteria (Li et al., 2019b).

In addition, iron trimesate nanoMOFs MIL-100 (Fe) (MIL stands for Material from Institut Lavoisier) exhibited high drug loading capacity soaking a variety of drugs from their aqueous solutions with yields close to $100 \%$. In the case of Gemcitabinemonophosphate (Gem-MP), the drug payload reached $\sim 30$ wt $\%$ with $>98 \%$ drug loading efficiency (Rodriguez-Ruiz et al., 2015). Gem-MP, the active intermediate of Gem, is widely used in various carcinomas, including pancreatic cancer, bladder cancer, and non-small cell lung cancer. The administration of Gem-MP is of high interest for resistant cancer treatment since the phosphorylation of Gem can be a rate-limiting step especially difficult for resistant cancer cells. However, Gem-MP administration is hampered by its poor stability in biological media and low cellular uptake (Bouffard et al., 1993). In this challenging context, some of us showed that Gem-MP could be protected against degradation with increased cellular uptake by encapsulation in nanoMOFs (Rodriguez-Ruiz et al., 2015).

Surface modifications are essential to control drug release and modulate the in vivo fate of nanoMOFs in the living body. Silica coatings were employed in an attempt to control the release of loaded molecules from nanoMOFs MIL-101 (TaylorPashow et al., 2009). NanoMOFs were coated with lipid bilayers to improve their uptake by cancer cells (Wuttke et al., 2015) or with chitosan to increase their intestinal permeability (Hidalgo et al., 2017). Heparin coatings endowed the nanoMOFs with longer-blood circulation time (Bellido et al., 2015).

Poly(ethylene glycol) (PEG) based materials remain the most employed ones to engineer coatings able to prevent nanoparticles (NPs) clearance by the innate immune system, which is a prerequisite for biomedical applications (Gref et al., 1994, 1995). However, as compared to dense polymeric NPs, the porous nanoMOFs are more challenging to be coated with PEG, because these linear chains readily penetrate within their porosity, inducing an uncontrolled "burst" drug release (Agostoni et al., 2015). There are still scarce examples of successful PEGylated nanoMOF formulations. PEG was crosslinked onto the nanoMOF's surface to avoid its penetration inside the porous cores (Giménez-marqués et al., 2018) but resulted in a non-biodegradable coating. Alternatively, nanoMOFs were coated with inclusion complexes consisting of functionalized

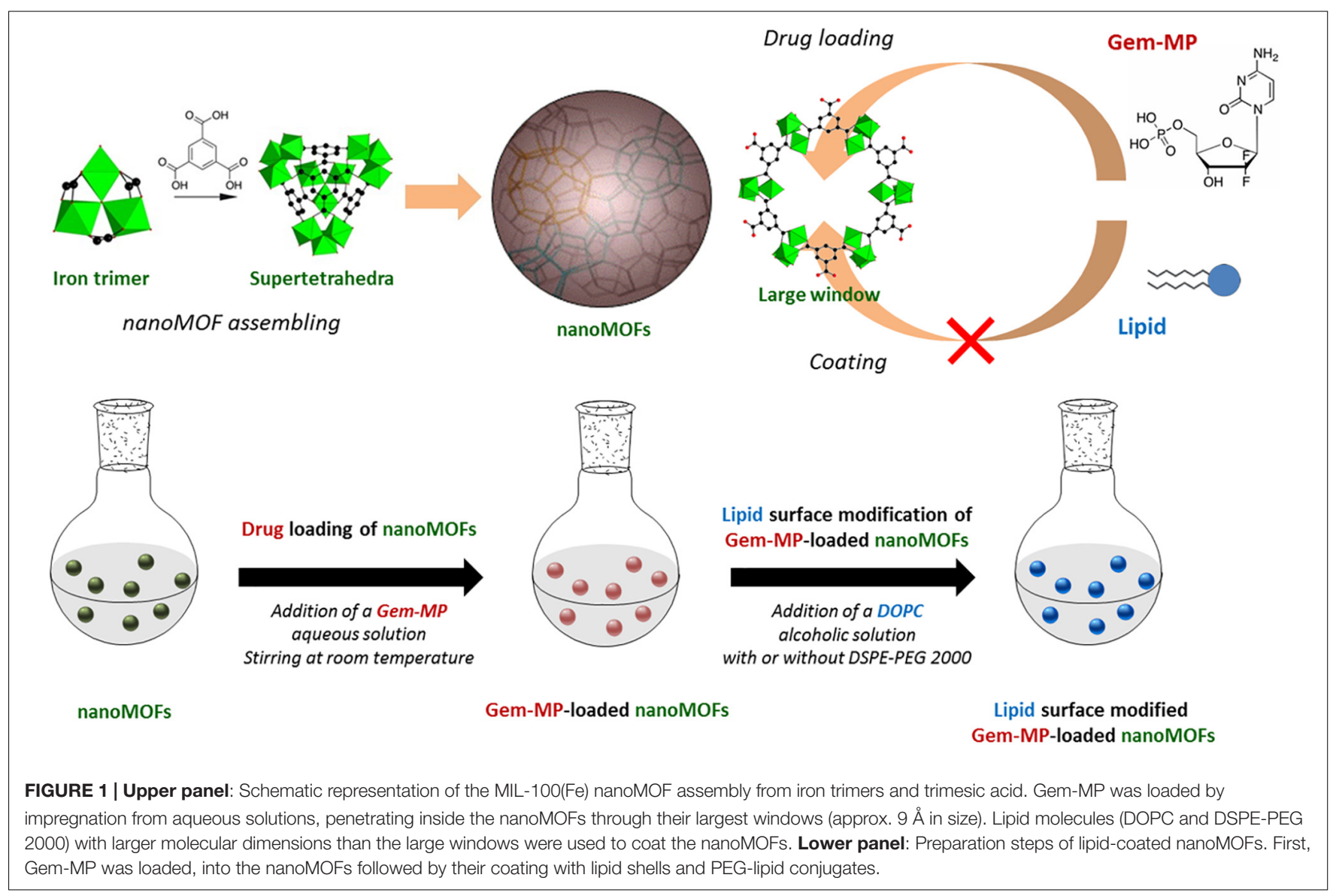


cyclodextrins (CDs) and PEG chains coupled to adamantine (Agostoni et al., 2015; Aykac et al., 2017; Cutrone et al., 2019a). Finally, comb-like copolymers consisting of polysaccharides grafted with moieties able to coordinate to the nanoMOFs and PEG chains were synthesized and anchored onto the nanoMOFs (Cutrone et al., 2019b). However, all these coatings imply sophisticated chemistry strategies and/or several preparation steps, which might restrict their further applications. Moreover, all the PEGylated (macro) molecules used in the previous studies are not approved by Food and Drug Administration (FDA).

In this context, we propose to engineer for the first time PEGylated coatings on nanoMOFs by using only FDAapproved materials using a convenient one-step method. To date, Doxil R and Onivyde R represent the only FDA-approved PEGylated NPs (Barenholz, 2012), where DSPE-PEG 2000 (1,2distearoyl-sn-glycero-3-phosphoethanolamine- $\mathrm{N}$-[amino (polyethylene glycol)-2000] sodium salt) were used in both cases. Herein, DSPE-PEG 2000 was used in combination with DOPC (1,2-dioleoyl-sn-glycero-3-phosphocholine) to functionalize the surface of iron trimesate MOFs (Figure 1 lower panel). Moreover, we show that the PEG-based coating have an impact on both drug release and nanoMOFs degradation, which was not the case with the coatings used so far. Finally, the coatings were able not only to reduce macrophage uptake in vitro but also to kill cancer cells.

\section{MATERIALS AND METHODS}

\section{Materials}

Iron (III) chloride hexahydrate (98\%) was purchased from Alfa Aesar (Schiltigheim, France). 1,3,5-benzenetricarboxylic acid (BTC, 95\%) and absolute ethanol (99\%) were from SigmaAldrich (Saint-Quentin-Fallavier, France). These materials were used for the synthesis of nanoMOFs. Amoxicillin (Amox) from Sigma-Aldrich (Saint-Quentin-Fallavier, France) and 2', $2^{\prime}$ difluorodeoxycytidine monophosphate (Gem-MP) from Toronto Research Chemicals (North York, Canada) were the drugs used in this study. 1,2-dioleoyl-sn-glycero-3-phosphocholine (DOPC) and (1,2-distearoyl-sn-glycero-3-phosphoethanolamine-N[amino(polyethylene glycol)-2000] sodium salt (DSPE-PEG 2000) were ordered from Avanti Polar Lipids (Alabama, United States) as coating materials. 3-(4,5-Dimethyl-2thiazolyl)-2,5-diphenyl-2H-tetrazolium bromide (MTT, Sigma-Aldrich, Oslo, Norway) was used for toxicity evaluation of nanoMOFs. All the chemicals were used without further purification.

\section{Cell Culture}

Murine macrophage cell line J774A.1, CelluloNet biobank BB0033-00072, were grown in RPMI-1640 medium (Thermo Fisher Scientific, Villebon-sur-Yvette, France) supplemented with $10 \% \mathrm{v} / \mathrm{v}$ decomplemented fetal bovine serum (FBS, Thermo Fisher Scientific, Villebon-sur-Yvette, France), 1\% L-Gluthamine (Sigma-Aldrich, Oslo, Norway), and 1\% (P/S, Sigma-Aldrich, Oslo, Norway) at $37^{\circ} \mathrm{C}$ in humidified air containing $5 \% \mathrm{CO}_{2}$. SKOV3 ovarian cancer cell were cultivated in a RPMI-1640 media without phenol red supplemented with 10\% FBS, 5\%
L-Gluthamine and $5 \%$ penicillin/streptomycin $(\mathrm{P} / \mathrm{S})$ at $37^{\circ} \mathrm{C}$ in a $5 \% \mathrm{CO}_{2}$ humidified atmosphere.

\section{Synthesis and Characterization of MIL-100(Fe) NanoMOFs}

Iron trimesate nanoMOFs was synthesized by microwave assisted hydrothermal reaction as previously described [6]. Briefly, $20 \mathrm{~mL}$ of aqueous mixture containing $6.0 \mathrm{mM}$ of iron chloride hexahydrate and $4.02 \mathrm{mM}$ of trimesic acid (TA, 1,3,5-benzenetricarboxylic acid) was heated at $130^{\circ} \mathrm{C}$ for $6 \mathrm{~min}$ under stirring. The reaction was carried out with the power of $1600 \mathrm{~W}$ (Mars-5, CEM, United States). The as-synthesized nanoMOFs were harvested by centrifugation $(10,000 \mathrm{~g}, 15 \mathrm{~min})$ and washed with absolute ethanol to remove the excessive TA until the supernatant became colorless. NanoMOFs were stored in ethanol at room temperature for further usage at the concentration of $18.2 \mathrm{mg} / \mathrm{mL}$.

SEM images were acquired on a Zeiss SUPRA 55 VP field emission gun scanning electron microscope fitted with an EDAX EDS analytical system. It was set to a low voltage $(1 \mathrm{kV})$ and low current (a few pA) in order not to damage the samples and to avoid any conductive coating that could bother direct observation of the samples. Secondary electron type detector was used to record the images.

Dynamic light scattering (DLS) measurements were performed at $25^{\circ} \mathrm{C}$ on a Malvern Zetasizer Nano-ZS instrument at $90^{\circ}$ angle. The mean hydrodynamic diameter of the particles was determined in a diluted aqueous suspension at $50 \mu \mathrm{g} / \mathrm{mL}$.

Nanoparticle tracking analysis (NTA) was performed on Malvern NanoSight (LM10 Instrument, Malvern Instruments Ltd., Orsay, France), which combines a conventional optical microscope with a laser to illuminate the NPs in Brownian motion. It is used to individually follow nanoMOFs to gain insight into their size distribution and concentration.

Zeta potential (ZP) of nanoMOFs were measured at $25^{\circ} \mathrm{C}$ using a Zetasizer Nano-ZS instrument at different $\mathrm{pH}$ ranging from 3 to 10 . NanoMOFs was diluted to $100 \mu \mathrm{g} / \mathrm{mL}$ with $1 \mathrm{mM} \mathrm{KCl}$. Measured electrophoretic mobilities were converted to zeta potential values according to the Smoluchowski equation. Nitrogen sorption measurements were performed on a Micromeritics Instruments ASAP 2020 at 77 K. Samples were degassed at $100^{\circ} \mathrm{C}$ for $15 \mathrm{~h}$. BET surface area was calculated in the partial pressure range of $0.05-0.20 \mathrm{P} / \mathrm{P}_{0}$.

\section{Drug Encapsulation in NanoMOFs}

Drugs (Gem-MP and Amox) were loaded within nanoMOFs simply by impregnation of drug(s) aqueous solutions and nanoMOFs. Practically, nanoMOFs suspension $(1.0 \mathrm{mg})$ were centrifuged for $10 \mathrm{~min}$ at $10,000 \mathrm{~g}$ and re-suspended in $1 \mathrm{~mL}$ of aqueous drug solutions $(0.125 \sim 1 \mathrm{mg} / \mathrm{mL}$ for Amox and $0.08 \sim$ $0.2 \mathrm{mg} / \mathrm{mL}$ for Gem-MP) or water as a control. Different drug concentrations were used to optimize the drug encapsulation. After incubation at room temperature under gentle stirring for several hours ( $12 \mathrm{~h}$ for Amox and $4 \mathrm{~h}$ for Gem-MP), the nanoMOFs were recovered by centrifugation at $10,000 \mathrm{~g}$ for $10 \mathrm{~min}$. The non-encapsulated drug in the supernatant was 
quantified by adapting previously described High Performance Liquid Chromatography (HPLC) methods (Li et al., 2019a,b). Specifically, HPLC analysis was performed on an Agilent system using a tunable UV absorbance detector. The injection volume of AMOX was $10 \mu \mathrm{L}$ followed by eluant flow at a rate of $0.5 \mathrm{~mL} / \mathrm{min}$ through a C18 Silica column $(4.6 \times 250 \mathrm{~mm}$, $5 \mu \mathrm{m}$; Phenomenex) maintained at $30^{\circ} \mathrm{C}$. The mobile phase consisted of $30 \%(\mathrm{v} / \mathrm{v})$ methanol containing $5.2 \mathrm{mg} / \mathrm{mL}$ of sodium dihydrogene phosphate monohydrate. The $\mathrm{pH}$ was adjusted to 5 using phosphoric acid solution. AMOX were detected at $247 \mathrm{~nm}$ and retention times were $4.6 \mathrm{~min}$. Similarly, Gem-MP was detected using the same Agilent system and column. The mobile phase was composed of $84 \%$ buffer [0.2 M (TEAA)]: $16 \%$ methanol. It was detected at $254 \mathrm{~nm}$ with an injection volume of $10 \mu$ l. The drug payload was calculated as Equation (1):

$$
\text { Payload }(\%)=\frac{\text { Encapsulated } \operatorname{Drug}(m g)}{\text { nanoMOFs }(m g)} \times 100
$$

\section{Surface Modification of NanoMOFs With DOPC Lipids and PEG-Lipid Conjugates}

Surface modification was performed using a "green" method. To prepare DOPC coated nanoMOFs, $60 \mu \mathrm{l}$ of nanoMOFs were mixed with $40 \mu \mathrm{l}$ of DOPC alcoholic solution containing $100 \mu \mathrm{g}$ of DOPC. Subsequently, $900 \mu \mathrm{l}$ of water were rapidly added using an electronic pipette. The weight ratio between DOPC and nanoMOF was in the range of 1:20 1:1. In the case of PEG-lipid conjugates coated nanoMOFs, $20 \mathrm{wt} \%$ of DOPC was replaced by DSPE-PEG 2000.

\section{Characterization of Lipid Coated NanoMOFs}

\section{Lipid Quantification}

DOPC quantification was performed by a colorimetric, enzymatic method (BIOLABO, Maizy, France) which is commonly used to determine the phospholipid amount in serum. This titration is based on the assay of the choline moiety of phospholipids. To do this, $10 \mu \mathrm{L}$ of specimens or a standard solution were mixed with the reagents in the BIOLABO titration kit. The mixtures were stirred $10 \mathrm{~min}$. at $37^{\circ} \mathrm{C}$. Then, the absorbance at $500 \mathrm{~nm}$ of all samples was measured. The DOPC concentration was finally calculated as Eq. (2):

$$
\begin{aligned}
& \text { DOPC concentration }= \\
& \text { Standard concentration } \times \frac{\text { Abs }(\text { specimen })}{\text { Abs }(\text { standard })} \times 100
\end{aligned}
$$

\section{NPs Concentration Measurements by NP Tracking Analysis (NTA)}

The concentration of nanoMOFs modified with DOPC or PEGlipid conjugates at different weight ratios was investigated by Nanosight (LM10 Instrument, Malvern Instruments Ltd., Orsay, France), which combines a conventional optical microscope with a laser to illuminate the NPs in Brownian motion. Of main interest here, the size distribution and concentration could be determined simultaneously. Results are expressed as means of five independent measurements.

\section{Colloid Stability Characterization by DLS}

The colloid stability of the nanoMOFs before and after lipid surface modification was monitored in water every day during 3 weeks' storage at $4^{\circ} \mathrm{C}$. The stability in biological medium, including cell culture medium and phosphate buffer saline (PBS) used in this study, was also measured at $0,0.5,1,2,4,6$, and $8 \mathrm{~h}$ after incubation at $37^{\circ} \mathrm{C}$.

\section{Drug Release and Degradation of nanoMOFs}

Drug release was performed in PBS of different concentrations at $37^{\circ} \mathrm{C}$. Briefly, drug loaded nanoMOFs were centrifuged at $10,000 \mathrm{~g}$ for $10 \mathrm{~min}$ and the pellet was re-dispersed in $1 \mathrm{~mL}$ water by vortex. Aliquots of $100 \mu \mathrm{L}$ were taken and mixed with $900 \mu \mathrm{l}$ of the media used for release. The final concentration of PBS was 1, 3, and $6 \mathrm{mM}$ and nanoMOFs of $2.0 \mathrm{mg} / \mathrm{mL}$. After different incubation times $(30 \mathrm{~min}, 1 \mathrm{~h}, 2 \mathrm{~h}, 4 \mathrm{~h}, 6 \mathrm{~h}$ and $24 \mathrm{~h}$ ), the suspensions were centrifuged and the supernatants were assessed by HPLC as previously described to determine the amount of released drug. Moreover, the trimesate release was also evaluated by HPLC. Briefly, trimesate was analyzed with a mobile phase consisting of $90 \%$ buffer $\left(5.75 \mathrm{~g} / \mathrm{L}\right.$ of $\left.\mathrm{NH}_{4} \mathrm{H}_{2} \mathrm{PO}_{4}\right): 10 \%$ Acetonitrile containing $5 \mathrm{mM}$ TBAP. The injection volume was $5 \mu \mathrm{l}$ and the detection wavelength was set at $220 \mathrm{~nm}$.

\section{Human Plasma Protein Adhesion Tests}

Human serum albumin (HSA) was used in this study. NanoMOFs modified or not $(300 \mu \mathrm{g} / \mathrm{mL})$ were incubated with HSA at $100 \mu \mathrm{g} / \mathrm{mL}$ in $10 \mathrm{mM}$ phosphate buffer at $37^{\circ} \mathrm{C}$. The samples were centrifuged at $10,000 \mathrm{~g}$ for $5 \mathrm{~min}$ to remove the nanoMOFs after 1, 2, 4, 6, 8 and $12 \mathrm{~h}$ incubation. The excessive amounts of HSA in the supernatant were quantified using a bicinchoninic acid (BCA) assay.

\section{NanoMOF Internalization in Macrophage}

NanoMOF internalization was quantified by Inductively Coupled Plasma Mass Spectrometry (ICP-MS). Macrophage cells (J774A.1) were seeded at a density of $2.0 \times 10^{5}$ cells per well in 24 -well plates. Cells were cultured at $37^{\circ} \mathrm{C}$ in $5 \% \mathrm{CO}_{2}$ overnight for attachment. Cells were then incubated with $1 \mathrm{~mL}$ cell culture media containing nanoMOFs coated or not with lipids (nanoMOF concentration $=50 \mu \mathrm{g} / \mathrm{mL}$ ). At the end of the $4 \mathrm{~h}$ incubation, the cells were washed with PBS for three times to eliminate the excess of MOFs. Cells were finally dried and digested using aqua regia (15 min under ultrasonic bath), Fe quantification was performed using an ICP-MS equipped with a triple quadrupole (Agilent 8800, Agilent Technologies, Japan). Fe and Co were added as internal standard on samples and calibration standards solution at a concentration of $10 \mu \mathrm{g} / \mathrm{L}$. Isotopes were detected using "on-mass mode" $\left({ }^{54} \mathrm{Fe}^{+},{ }^{56} \mathrm{Fe}^{+}\right.$, ${ }^{59} \mathrm{Co}^{+}$). Helium was introduced into the collision/reaction cell at a flow rate of $3 \mathrm{~mL} / \mathrm{min}$. Dwell time for each of the targeted isotopes was $1 \mathrm{~s}$. Fe was quantified using external calibration prepared using certified $1000 \mathrm{mg} / \mathrm{L}$ Fe standard solution (Merck, Germany). Operation conditions were daily optimized using a tuning solution. 


\section{Cytotoxicity Assessment}

MTT assays were carried out on SKOV3 ovarian cancer cell line to investigate the cytotoxicity of NPs. The cells were plated in 96 well plates at a concentration of 10,000 cells per well. The media was removed after $24 \mathrm{~h}$ incubation and replaced by fresh media containing the MOFs nanoparticles at different concentrations. The cytotoxicity was assessed by MTT assay at 24, 48 and $72 \mathrm{~h}$ following the incubation of the cells with the MOFs. In brief, $100 \mu \mathrm{L}$ of complete media containing $0.5 \mathrm{mg} / \mathrm{mL}$ of MTT were added to cells and incubated for $2 \mathrm{~h}$ at $37^{\circ} \mathrm{C}$ in a $5 \%$ $\mathrm{CO}_{2}$ humidified atmosphere. Subsequently, the MTT media were removed and replaced by $100 \mu \mathrm{L}$ of DMSO per well to dissolve the MTT-formazan crystals. The plates were shaken for $10 \mathrm{~min}$ at $350 \mathrm{rpm}$ in a Heidolph Titramax 101 orbital shaker, and the absorbance at $595 \mathrm{~nm}$ was measured with the Tecan spark M10 plate reader. Each MTT experiment was reproduced three times.

\section{RESULTS AND DISCUSSION}

\section{MIL-100 (Fe) nanoMOF Surface Modification and Characterization of Functionalized NanoMOFs}

Iron trimesate nanoMOFs with mean diameters of $232 \pm 14 \mathrm{~nm}$ and Brunauer-Emmett-Teller (BET) surface areas of $1519 \pm 50 \mathrm{~m}^{2} \cdot \mathrm{g}^{-1}$ were successfully synthesized by a "green" organic solvent-free hydrothermal method exempt of toxic additives such as hydrofluoric acid (Agostoni et al., 2013). They were crystalline and exhibited a facetted morphology (Figure 2A) in agreement with previously reported data (Cutrone et al., 2019a,b).

In an attempt to achieve "stealth" NPs, the as-synthesized nanoMOF were surface functionalized with PEG-lipid conjugates in a one-step procedure using a mixture of DSPE-PEG 2000 and DOPC. Lipids were associated within less than $2 \mathrm{~min}$ at room temperature by dispersing the nanoMOFs in an ethanolic aqueous solution containing both DSPE-PEG 2000 and DOPC, followed by a quick addition of water to favor lipid deposition onto the nanoMOF surface. Indeed, lipids were freely soluble in ethanol/water mixtures, but they readily precipitated upon progressive addition of water which drastically reduced their solubility, leading to precipitation onto the nanoMOF surfaces (Wuttke et al., 2015). DOPC-coated nanoMOFs were prepared as controls using the same method. The bare and coated nanoMOFs were characterized by a set of complementary methods.

Firstly, SEM images show that the lipid-coated nanoMOFs displayed similar shapes but with more rounded edges (Figure 2B) as compared to the uncoated ones (Figure 2A), possibly because surface modification. No significant differences were observed for the coated nanoMOFs with or without PEG-lipid conjugates (Figure 2C). Secondly, EDX experiments were performed to detect the presence of elements specific to the MOF cores $(\mathrm{C}, \mathrm{O}, \mathrm{Fe})$ and to the shells $(\mathrm{C}, \mathrm{O}, \mathrm{N})$ in the top layers of the NPs (around $10 \mathrm{~nm}$ depth). The presence of the DOPC coating was evidenced by the detection of an $\mathrm{N}$ peak characteristic of DOPC which was not found with bare nanoMOFs (Supplementary Figure S1). Interestingly, in the PEG shells obtained with the lipid mixtures, the relative $\mathrm{O}$ content was increased by a factor of 4 as compared to DOPC coatings (Supplementary Figure S1) possibly due to the presence of PEG chains in the nanoMOFs' top layers, as PEG has the highest $\mathrm{O}$ content from all the nanoMOF components. These data offer a straightforward proof for both the presence of DOPC and PEG-lipid conjugates in the nanoMOF top layers.

The amount of DOPC in the nanoMOFs was quantified by using a colorimetric enzymatic method. For this, the DOPC:nanoMOF weight ratio in the preparation procedure was varied from 1:20 to 1:1. As shown in Figure 3A, the amount of lipids associated to the nanoMOFs increased with the amount of lipids used in the coating procedure. A plateau was reached at a DOPC: nanoMOF weight ratio of 1:3, corresponding to $25 \pm 4$ $w t \%$ lipids associated to the nanoMOFs. These quantities of coating material are among the highest reported so far (Horcajada et al., 2010; Agostoni et al., 2015; Bellido et al., 2015; Hidalgo et al., 2017; Giménez-marqués et al., 2018; Cutrone et al., 2019a,b). As comparison, phosphorylated cyclodextrin (CD-P) coatings on same iron trimesate nanoMOFs reached $\sim 17 \mathrm{wt} \%$ (Agostoni et al., 2015). The important lipid association could be possibly due to: i) the fast precipitation of lipids at the hydrophobic surface of nanoMOFs, and ii) the strong affinity of the phosphate groups in the lipids for the iron sites at the nanoMOFs' surface.

The nanoMOFs, coated or not, were characterized by a set of complementary methods. First, X-ray powder diffraction
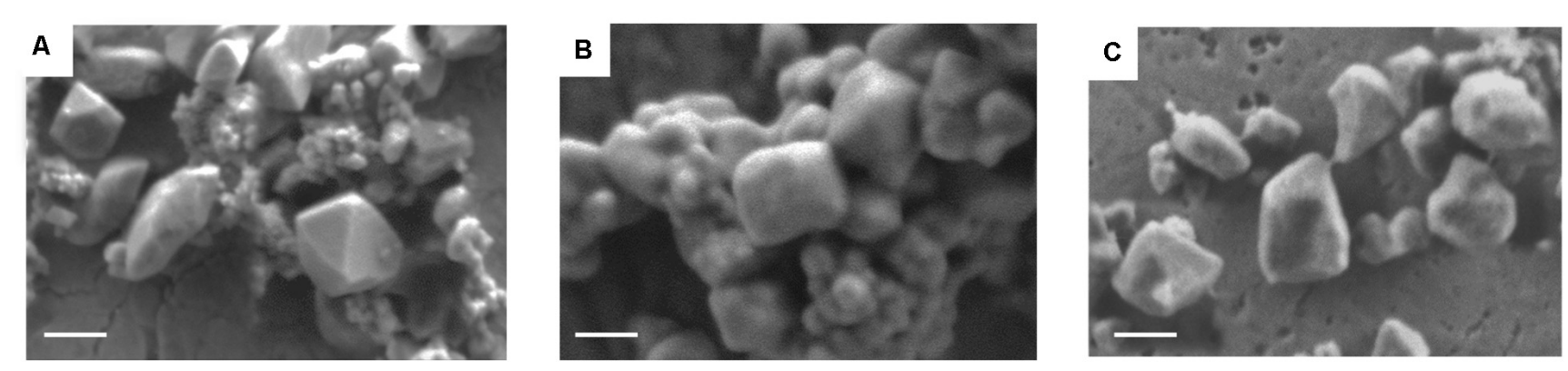

FIGURE 2 | Representative scanning electron microscope (SEM) images of nanoMOFs before and after lipid modification. (A) nanoMOFs; (B) nanoMOFs after modification with DOPC; (C) nanoMOFs after modification with DOPC and DSPE-PEG 2000. Scale bar: $100 \mu \mathrm{m}$. 

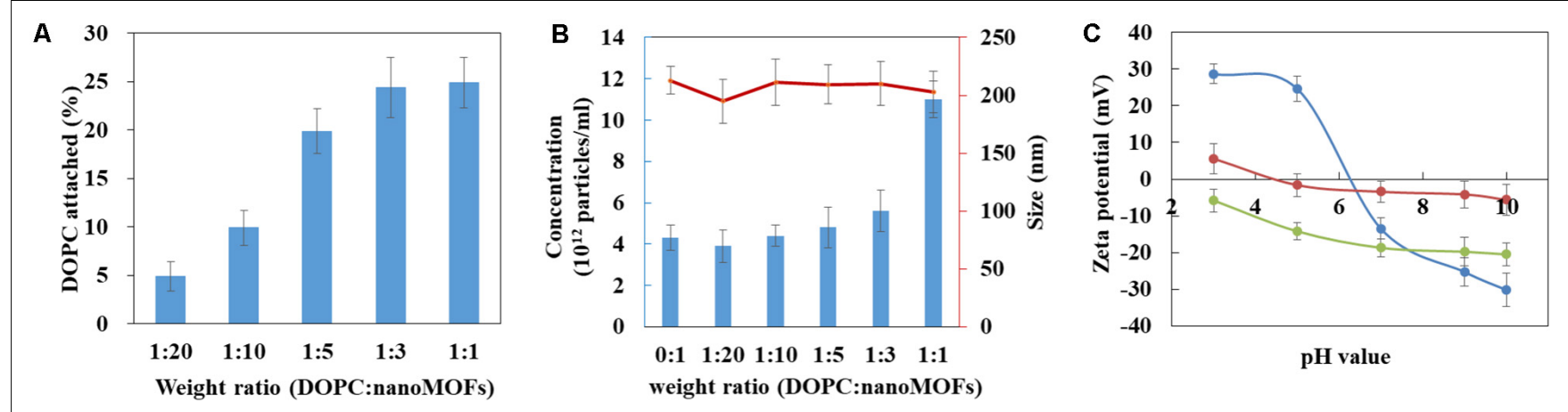

FIGURE 3 | Characterization of lipid-coated nanoMOFs. (A) Quantification of the amount of DOPC in the nanoMOFs; (B) Mean hydrodynamic diameter (red) and concentration (blue) of DOPC-coated nanoMOFs determined by NTA. (C) Zeta potential of nanoMOFs as a function of pH before (blue) and after lipid coating with (red) or without (green) the addition of DSPE-PEG 2000.

(XRPD) showed that the crystalline structure of the nanoMOFs was preserved after surface modification (Supplementary Figure S2). Dynamic light scattering (DLS) proved that there were no significant differences between the mean hydrodynamic diameters of nanoMOFs before and after surface functionalization $(232 \pm 14 \mathrm{~nm}, 241 \pm 17 \mathrm{~nm}$ and $238 \pm 11 \mathrm{~nm}$ for uncoated nanoMOFs, lipid coated nanoMOFs at a DOPC: nanoMOF weight ratio of $1: 3$, with and without DSPE-PEG 2000, respectively). Moreover, the BET surface areas were not affected by surface modification with lipids $\left(1519 \pm 50 \mathrm{~m}^{2} \cdot \mathrm{g}^{-1}\right.$, $1486 \pm 70 \mathrm{~m}^{2} \cdot \mathrm{g}^{-1}$, and $1547 \pm 80 \mathrm{~m}^{2} \cdot \mathrm{g}^{-1}$ for uncoated nanoMOFs, lipid coated nanoMOFs with and without DSPEPEG 2000, respectively), suggesting that the bulky lipids were located onto the nanoMOF's external surfaces rather than into their porosity.

Before surface modification, the nanoMOF concentration was around $(4 \pm 0.8) \times 10^{12}$ particles $/ \mathrm{mL}$, as determined by Nanoparticle Tracking Analysis (NTA). Interestingly, the nanoMOF particle concentration did not change upon modification with lipids (Figure 3B, blue histograms), suggesting that the lipids adhered at their surface and did not remain into the suspension medium. Indeed, at DOPC:nanoMOFs weight ratios from $0: 1$ up to $1: 3$, both particle concentrations and mean hydrodynamic diameters were unaffected $\left[(5.6 \pm 0.8) \times 10^{12}\right.$ particles $/ \mathrm{mL}$, and $210 \pm 23 \mathrm{~nm}$, respectively]. To support this hypothesis, DLS analysis of supernatants (Supplementary Table S1) after particle centrifugation revealed that they were devoid of any lipid vesicles ( $<1 \%$ particles free).

However, addition of excess lipids (weight DOPC:nanoMOF ratio of $1: 1)$ resulted in a dramatic increase of total particle concentration [from $(4 \pm 0.8) \times 10^{12}$ to $(1.1 \pm 0.4) \times 10^{13}$ particles $/ \mathrm{mL}$ ], presumably because the nanoMOF surfaces were saturated with lipids. Of note, the mean hydrodynamic diameter of the nanoMOFs was unaffected, only the polydispersity index (PdI) increased from 0.15 to 0.25 , possibly because of the presence of lipid vesicles in excess. Note that the association of DSPE-PEG 2000 didn't significantly influence the mean hydrodynamic diameter, nor the nanoMOF's concentration (less than $10 \%$ variations) suggesting that the PEGylated lipids also attached onto the nanoMOFs. In conclusion, lipids were associated up to $25 \pm 4 \mathrm{wt} \%$ without inducing any changes in nanoMOF porosities, size distribution, and crystallinity.

Interestingly, the presence of the coatings affected the nanoMOFs electrophoretic mobility, as shown by Zeta potential (ZP) investigations in Figure 3C. Indeed, the $\mathrm{ZP}$ of the uncoated nanoMOFs was strongly dependent upon the $\mathrm{pH}$ of the suspension medium, shifting from positive values $(+23 \pm 3$ $\mathrm{mV})$ at $\mathrm{pH}$ lower than 5 to negative values $(-15 \pm 3 \mathrm{mV})$ at basic $\mathrm{pH}$. This could be probably due to the presence of both uncoordinated iron sites and terminal carboxyl groups of the trimesate ligands at the external nanoMOFs surface (Cutrone et al., 2019b). The ZP values were dramatically altered after surface modification (Figure 3C). DOPC-coated nanoMOFs displayed negative $\mathrm{ZP}$ values $(-6$ to $-20 \mathrm{mV}$ ) whatever the $\mathrm{pH}$ in the range of 3 to 10 , in line with data reported for DOPC liposomes (Chibowski and Szcześ, 2016). These results support the presence of DOPC lipid layers onto the nanoMOFs which shield their charged surface moieties. Interestingly, when the nanoMOFs were surface-functionalized with PEG chains, their $\mathrm{ZP}$ values were shifted to neutral $(-1.6 \pm 3.4 \mathrm{mV})$. This is in good agreement with other studies on PEG-coated NPs (Gref et al., 1995; Thevenot et al., 2007; Troutier and Ladavière, 2007; Troutier-Thuilliez et al., 2009; Bugnicourt et al., 2019).

\section{Effect of the Coatings on the Colloidal Stability of NanoMOFs in Biological Media}

As the majority of uncoated NPs, nanoMOFs suffer from poor stability in biological media, which hampers their biomedical applications. Figure 4 clearly shows that uncoated nanoMOFs undergo a fast aggregation in both phosphate buffer saline (PBS, $\mathrm{pH}=7.4,10 \mathrm{mM}$ ) and cell culture medium DMEM (Dulbecco's Modified Eagle Medium) without fetal bovine serum (FBS), with the mean hydrodynamic diameters rapidly increasing to more than $1 \mu \mathrm{m}$ within only $6 \mathrm{~h}$ at $37^{\circ} \mathrm{C}$ (Figure 4). No significant variation was observed for the mean hydrodynamic diameter of uncoated nanoMOFs in water in the first $1 \mathrm{~h}$, however, they tended to aggregate upon storage (Supplementary Figure S3). They were stable only in DMEM supplemented with $10 \%$ (v/v) 


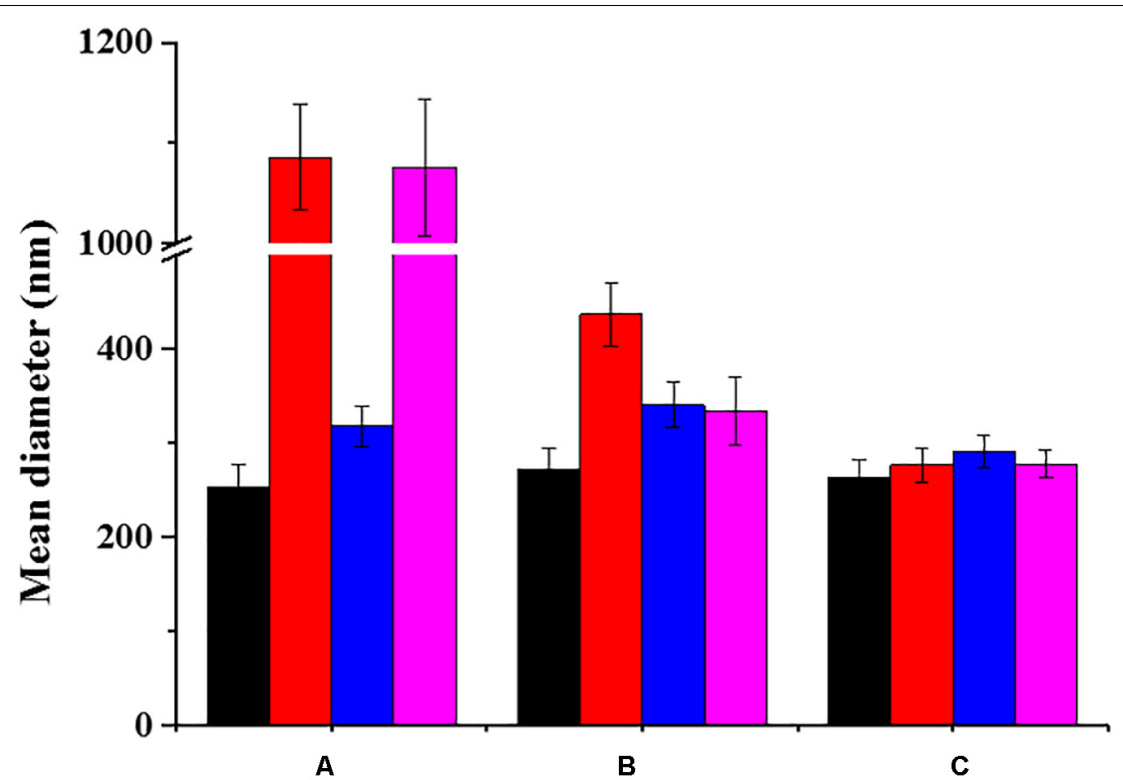

FIGURE 4 | Colloidal stability of nanoMOFs in different media, before (A) and after surface functionalization with DOPC (B) or PEG-lipid conjugates (C). Mean hydrodynamic diameters of nanoMOF suspensions at $100 \mu \mathrm{g} / \mathrm{mL}$ were determined by DLS after 6 h incubation at $37^{\circ} \mathrm{C}$. (Black: water; red: PBS; blue: DMEM supplemented with 10v/v\% FBS; pink: DMEM without FBS).

FBS, possibly due to the formation of a protein corona at their surface preventing their aggregation (see section "Effect of surface functionalization of nanoMOFs on protein adsorption and macrophage uptake").

In contrast, DOPC coated nanoMOFs were stable both in water and DMEM. No aggregation was observed even after 3 weeks storage. However, they still underwent aggregation in PBS (Figure 4). Remarkably, PEGylation allowed circumventing stability issues, whatever the suspension media (less than $10 \%$ diameter variation in PBS).

As all the coated and uncoated nanoMOFs were stable in DMEM supplemented with $10 \%$ (v/v) FBS, it was possible to explore further their cytotoxicity and interactions with cancer cell lines and macrophages. The PEGylated nanoMOFs exhibited excellent colloidal stability in all the tested biological media and thus appeared as optimal candidates for biological applications.

\section{Control of Degradation and Drug Release by Lipid Coating}

There is a general agreement on the fact that once the nanomaterials release their drug cargo, they should degrade to avoid accumulation inside the body (Horcajada et al., 2012). However, Fe-based nanoMOFs are reported to degrade rapidly in the biological media, because of coordination of various ions (phosphates, sulfates, etc.) to their iron sites, sometimes leading to uncontrolled "burst" drug release (Agostoni et al., 2013; Li et al., 2017, 2019b). It was therefore interesting to investigate if the hydrophobic lipid coatings could interfere with the rapid penetration of the aqueous degrading media inside the pores, thus allowing gaining better control upon the release and degradation mechanisms.
Degradation of nanoMOFs is generally monitored by the release of the constituting ligand trimesate (Agostoni et al., 2013; Rodriguez-Ruiz et al., 2015; Li et al., 2017). The degradation of the lipid-coated or bare nanoMOFs was studied by assaying ligand trimesate by HPLC in PBS (Figure 5A). In PBS $1 \mathrm{mM}$, uncoated nanoMOFs (blue curve, Figure 5A) underwent a fast degradation in the first $1 \mathrm{~h}$ at $37^{\circ} \mathrm{C}$ with around $15.5 \pm 1.1 \%$ trimesate released, in agreement with previous reports (Li et al., 2017). It was discovered that in the same conditions, the lipid-coated nanoMOFs, with (red curve, Figure 5A) or without PEG-lipids conjugates (green curve, Figure 5A), exhibited much slower degradation profiles than the uncoated nanoMOFs, with only $10 \pm 0.2 \%$ trimesate release in the first $1 \mathrm{~h}$. This suggests a more progressive diffusion of the phosphate ions into the coated nanoMOFs, slowing down their degradation. However, the same plateau was reached after $24 \mathrm{~h}$ incubation, corresponding to a total complexation of the phosphates in the medium (Li et al., 2017). In conclusion, the shell efficiently delayed the degradation process.

Then, the effect of lipid coatings on drug release was studied. Selected drug of interest was Gem-MP, a hydrophilic drug with low cell permeability. NanoMOFs acted as efficient "nanosponges", soaking Gem-MP from their aqueous solution with almost perfect efficiency (>98\%). Maximal loadings reached $25 \mathrm{wt} \%$ reflecting the strong interaction between the drug and the iron trimesate matrices. Advantageously, the lipid coating process didn't induce any significant drug release (less than 3\% variations before and after coating).

Gem-MP release is governed by a competition of coordination between the phosphate moieties in Gem-MP and free phosphates in PBS for the iron(III) Lewis acids 

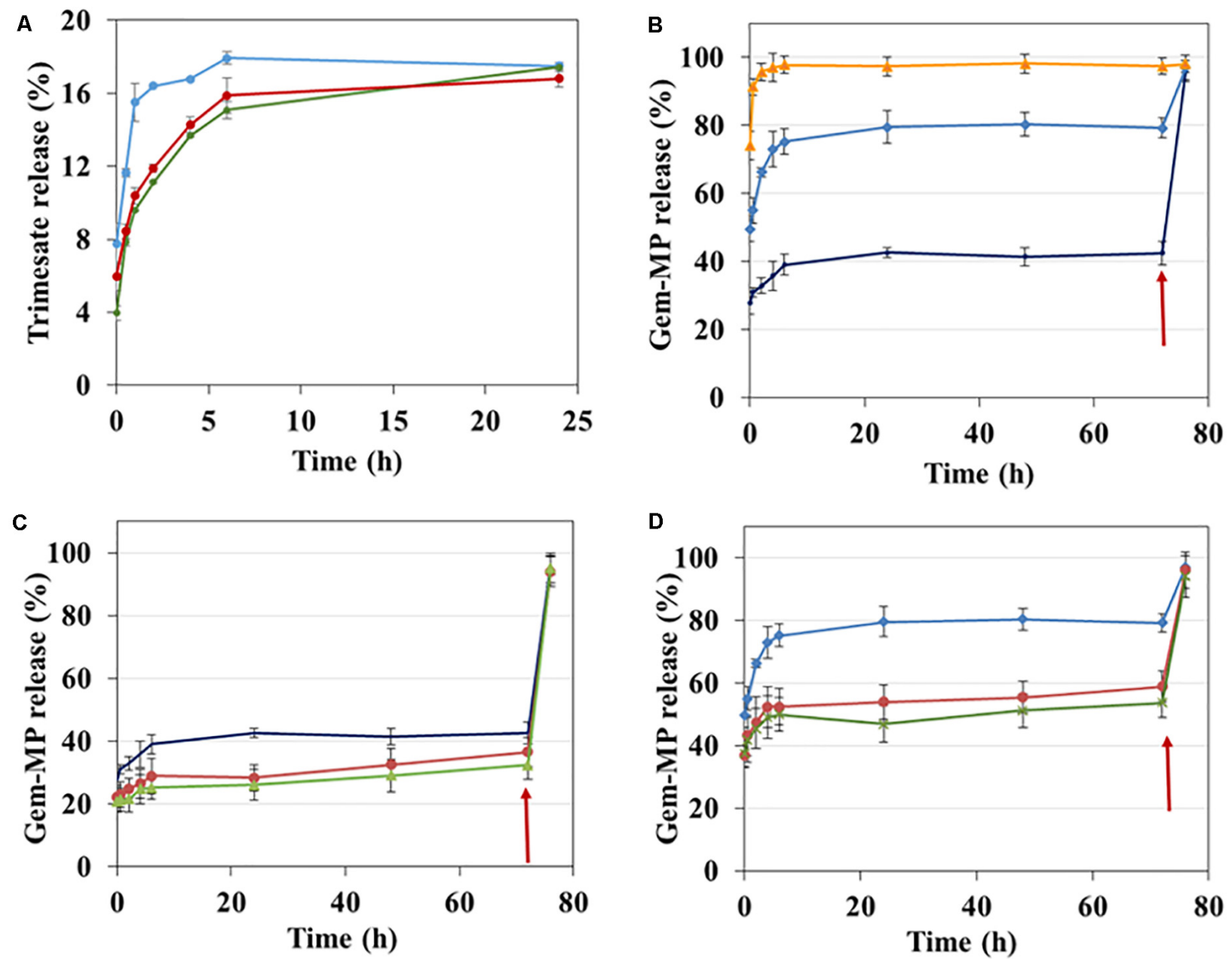

FIGURE 5 | Effect of surface modification on Gem-MP and trimesate release analyzed by HPLC. (A) Trimesate release in 1 mM PBS from nanoMOFs before (blue) and after lipid coating with (red) or without (green) coating with PEG-lipid conjugates after incubation at $37^{\circ} \mathrm{C}$. (B) Gem-MP release from uncoated nanoMOFs in PBS with different molarities (orange: $6 \mathrm{mM}$, blue: $3 \mathrm{mM}$; dark blue: $1 \mathrm{mM}$ ). Gem-MP release in $1 \mathrm{mM}$ (C) or 3 mM (D) PBS from nanoMOFs before (blue/dark blue) and after lipid coating with (red) or without (green) coating with PEG-lipid conjugates. In all cases (B-D), phosphate concentration was adjusted to $10 \mathrm{mM}$ after $72 \mathrm{~h}$ incubation at $37^{\circ} \mathrm{C}$ (red arrows), followed by further incubation for $4 \mathrm{~h}$ at $37^{\circ} \mathrm{C}$.

of nanoMOFs (Agostoni et al., 2013, 2015; Rodriguez-Ruiz et al., 2015). As expected, it was found that the higher the amount of phosphates, the higher the amount of drug released (Figure 5B). At low phosphate concentrations (PBS $1 \mathrm{mM}$ or $3 \mathrm{mM}$ ), a plateau (around $40 \%$ or $80 \% \mathrm{Gem}$ MP release) was reached in $24 \mathrm{~h}$, when all the phosphate molecules present in the release medium were complexed to the iron sites, as previously reported (Agostoni et al., 2013, 2015; Rodriguez-Ruiz et al., 2015). When additional phosphates were added in the release medium, all the drug still remaining in the nanoMOFs was immediately released (Figure 5B, arrow). Gem-MP release was well correlated with particle degradation, resulting in trimesate release (Supplementary Figure S4).

The presence of the lipid coating reduced the drug release from the nanoMOFs (Figure 5C). For instance, after $6 \mathrm{~h}$ incubation in PBS $1 \mathrm{mM}$, around $30 \% \mathrm{Gm}-\mathrm{MP}$ was released from the coated nanoMOFs, in comparison to $40 \%$ with the uncoated ones. This is possibly due to the restricted diffusion of phosphates into the nanoMOFs because of the lipid coating. Similarly, after $6 \mathrm{~h}$ incubation in PBS $3 \mathrm{mM}$, around 50\% Gem-MP was released from the coated nanoMOFs, in comparison to $78 \%$ with the uncoated ones (Figure 5D). The Gem-MP release from coated nanoMOFs gradually increased in a sustained manner in the following days (Figures 5C,D). All the remained drugs could be released out after $4 \mathrm{~h}$ incubation in concentrated phosphate buffer (10 mM PBS).

Similar results were found with another drug, amoxicillin (Amox). The amount of Amox released in the first hour of incubation release was reduced by a factor of two in the case of lipid-coated nanoMOFs as compared to the naked ones (Figure 6A), confirmed by different dilution factors at 10, 20, and 40 (Figure 6B). However, in the presence of strongly 
A

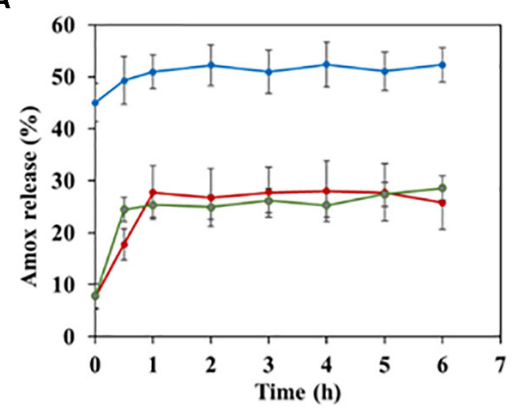

B

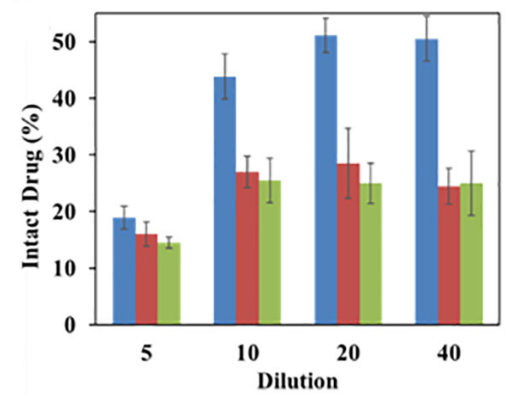

C

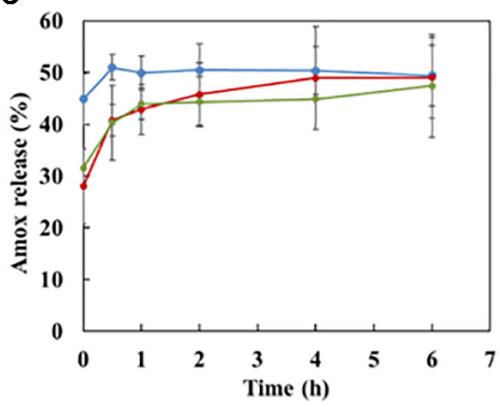

FIGURE 6 | Effect of coating on Amox release in water (A,B) and in PBS (C). (A) Release kinetics of Amox in water from nanoMOFs (1 mg/mL) before or after coating, with a dilution factor of 20; (B) Effect of dilution factor on Amox release after $4 \mathrm{~h}$ incubation at $37^{\circ} \mathrm{C}$ in water (Blue: uncoated nanoMOFs; red: DOPC coated nanoMOFs; Green: DOPC and PEG-lipid conjugate coated nanoMOFs).

complexing phosphates, the degradation was only delayed, but not avoided (Figure 6C).

\section{Cytotoxicity Assays of nanoMOFs on Ovarian Cancer Cells}

All the studied nanoMOFs were non-toxic for the SKOV3 ovarian cancer cells up to $100 \mu \mathrm{g} / \mathrm{mL}$ (Figure 7A, blue histograms), with more than $98 \%$ cell viability in $24 \mathrm{~h}$, which is in agreement with the previously reported lack of toxicity of these materials (Horcajada et al., 2010; Baati et al., 2013; Bellido et al., 2015; Giménez-marqués et al., 2018; Li et al., 2019b). In contrast, as expected, the anticancer drug Gem-MP $(20 \mu \mathrm{g} / \mathrm{mL}$, Figure 7A, brown histograms) exerted a cytotoxic effect with $45 \%$ cell viability after $48 \mathrm{~h}$ incubation, which further diminished to $29 \%$ in 72 h. Remarkably, Gem-MP loaded nanoMOFs showed a strong in vitro activity on SKOV3 ovarian cancer cells, higher than the free drug (Figure 7). At equivalent GemMP concentrations, whatever the drug loading (8 or $20 \mathrm{wt} \%$ ) and the amount of nanoMOF in contact with the cells (10 to $100 \mu \mathrm{g} / \mathrm{mL}$ ), the drug-loaded nanoMOFs outperformed the free drug in terms of toxicity on cancer cells (Figure 7 and Supplementary Figure S5).

This is in line with previous studies showing the efficient internalization of nanoMOFs bearing or not a lipid coating in pancreatic, breast, or bladder cancer cell lines (Rodriguez-Ruiz et al., 2015; Wuttke et al., 2015; Li et al., 2019a). It was recently shown that nanoMOFs acted as "Trojan horses" internalized by cancer cells, carrying their Gem-MP cargo to interfere with DNA (Li et al., 2019a). In this study it was shown that interestingly, the presence of a lipid coating (PEGylated or not) did not reduce the nanoMOF anticancer efficacy on SKOV3 ovarian cancer cells.

\section{Effect of Surface Functionalization of NanoMOFs on Protein Adsorption and Macrophage Uptake}

It is well known that intravenously administered NPs are readily covered by plasma proteins, creating the so-called "protein corona", which plays a crucial role on the NPs' biodistribution and in vivo fate (Gref et al., 2000). To gain insight on the influence of lipid coating of nanoMOFs on protein adsorption, HSA (human serum albumin), the most abundant protein in human blood plasma, was selected for this study.

NanoMOFs coated or not with DOPC lipids and PEG-lipid conjugates were incubated for $4 \mathrm{~h}$ at $37^{\circ} \mathrm{C}$ with HSA. After separation of the supernatants by centrifugation, the amount of non-adsorbed HSA was quantified using a BCA titration in order to determine the adsorbed HSA amounts onto nanoMOFs, lipid-modified or not. These amounts, expressed as $\mu \mathrm{g} / \mathrm{mg}$ of nanoMOFs, are reported in Figure 8A. In the case of uncoated nanoMOFs (Figure 8A, blue curve), the amount of adsorbed HSA reached a plateau within $6 \mathrm{~h}$, with around $50 \mu \mathrm{g} \mathrm{HSA} / \mathrm{mg}$ nanoMOFs. Interestingly, lipid coating dramatically reduced HSA adsorption, to only $\sim 5 \mu \mathrm{g} \mathrm{HSA} / \mathrm{mg}$ nanoMOFs (Figure 8A, green curve), regardless of the addition of DSPE-PEG 2000 (Figure 8A, red curve). To the best of our knowledge, these adsorbed HSA amounts are among the lowest reported with MIL100 (Fe) nanoMOFs (Gref et al., 2000; Cutrone et al., 2019b), suggesting that lipid-based coating on nanoMOFs is efficient to avoid albumin adsorption.

The potential "stealth" effect of the lipid-coated nanoMOFs, PEGylated or not, was evaluated on the murine macrophage cell line J774. Quantitative data on the amounts of nanoMOFs internalized by cells were obtained by ICP-MS, after extensive washing to remove the non-associated particles. An incubation time of $4 \mathrm{~h}$ was chosen as it corresponds to the typical blood circulation time of PEG-coated NPs (Cutrone et al., 2019b). Interestingly, the DOPC coating of nanoMOFs reduced their macrophage uptake by a factor of 2.4, from $75 \pm 6 \%$ to $31 \pm 3 \%$ (Figure 8B). The nanoMOF functionalization with PEG chains was even more effective, reducing their interactions with macrophage to $21 \pm 2 \%$. Despite these great in-vitro results, it is widely known that numerous complex interactions can occur after NP administration in multicellular organisms. Therefore, in vivo studies need to be carried on to demonstrate the efficacy of the PEG coating to reduce reticuloendothelial system (RES) uptake.

Nevertheless, it has to be noted that, in similar experimental conditions, other coating materials showed higher interactions with macrophages, for instance, $41 \pm 3 \%$ for CD-P coating, and 

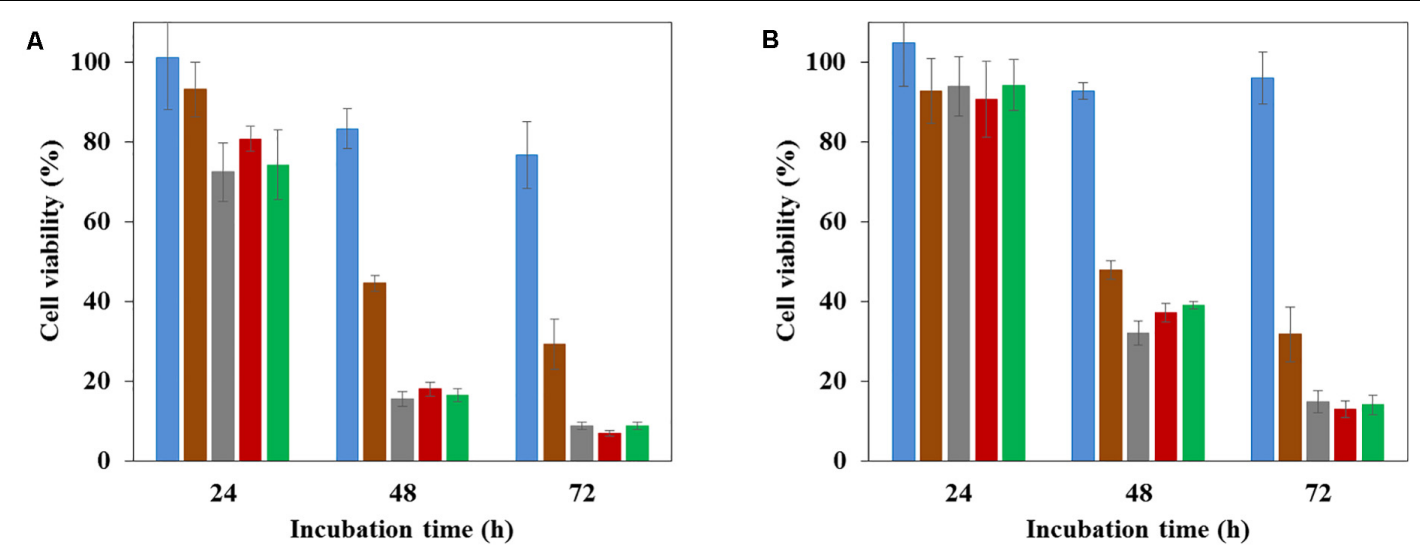

FIGURE 7 | Cytotoxcity measured by MTT assays of nanoMOFs (blue), anticancer efficacy of Gem-MP (brown), Gem-MP loaded nanoMOFs before (gray) and after coating with DOPC (red) or PEG-lipid conjugates (green). The experiments were performed on SKOV3 ovarian cancer cells, at different incubation times, with different concentrations nanoMOFs. (A) $100 \mu \mathrm{g} / \mathrm{mL}$; (B) $30 \mu \mathrm{g} / \mathrm{mL}$. Gem-MP loading was 20 wt\%.
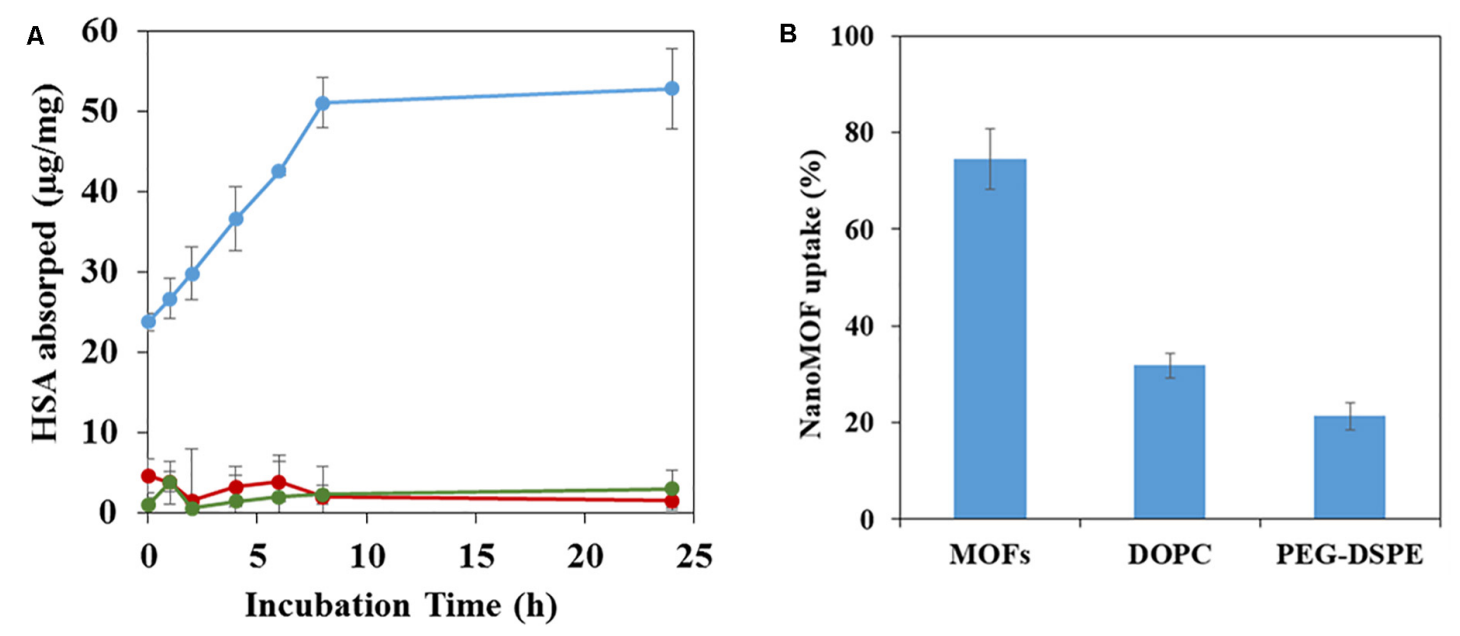

FIGURE 8 | HSA adsorption assayed by BCA assay (A) and $\mathbf{J 7 7 4}$ murine macrophage uptake (B) of nanoMOFs before and after lipid surface functionalization. (A) HSA adsorption by nanoMOFs before (blue) and after lipid coating with (red) or without (green) the addition of DSPE-PEG 2000. (B) NanoMOFs (before and after lipid coating, with or without addition of DSPE-PEG 2000) internalization inside murine macrophage $\mathrm{J774}$ cells. $50 \mu \mathrm{g} / \mathrm{mL}$ nanoMOFs were incubated with $3 \times 10^{5}$ $\mathrm{J} 774$ cells for $4 \mathrm{~h}$, and the amount of internalized nanoMOFs was determined by ICP-MS and expressed as a\% of the initial nanoMOF amount in contact with the cells. Significant difference was observed for nanoMOFs before and after surface modification $(p<0.01)$.

$39 \sim 24 \%$ for comb-like copolymers (Cutrone et al., 2019a,b). The advantage of lipid coating, demonstrated in this work, is a straightforward method, leading to efficient and stable coatings based on already FDA-approved materials. Lipid coatings on NPs are considered to be a promising strategy for the treatment of severe pathologies such as cancer (Luchini and Vitiello, 2019). In this study, the lipid coating not only afforded a control upon cell interaction but also provided a biocompatible protective barrier, modulating drug release and nanoMOF degradation. Of note, the nanoMOFs used in this study were shown to be biocompatible after intravenous administration in rats (Baati et al., 2013). However, the biocompatibility of the supermolecules assembled resulting from nanoMOFs coating with lipids has to be demonstrated in vivo.

\section{CONCLUSION}

The surface of iron trimesate nanoMOFs was successfully modified with FDA approved DSPE-PEG 2000 in combination with DOPC by a fast solvent-exchange deposition method. We described herein the preparation and comprehensive characterization of the lipid modified NPs. We showed, for the first time, that the lipid surface modification of porous nanoMOFs reduced their tendency to degrade rapidly in PBS. Moreover, the coating of nanoMOFs with PEG-lipid conjugates successfully decreased their uptake by macrophages in vitro by a factor of 3.6. Finally, nanoMOFs acted as "Trojan horses" internalizing inside the cancer cells, and carrying their Gem-MP cargo to interfere with DNA. 


\section{DATA AVAILABILITY STATEMENT}

The datasets generated for this study are available on request to the corresponding author.

\section{AUTHOR CONTRIBUTIONS}

RG conceived the study. RG, XL, and GS designed the experiments. XL, GS, and JQ preformed the experiments. CL contributed to the lipid investigations. $\mathrm{XL}$ and $\mathrm{RG}$ wrote the manuscript. MM, KB, and TT contributed to the biological evaluations. All authors approved the submitted version.

\section{FUNDING}

Financial support for this work was provided the French National Research Agency (ANR-14-CE08-0017 and ANR-16-CE18-0018)

\section{REFERENCES}

Agostoni, V., Chalati, T., Horcajada, P., Willaime, H., Anand, R., Semiramoth, N., et al. (2013). Towards an improved anti-HIV activity of NRTI via MetalOrganic Frameworks nanoparticles. Adv. Healthc. Mater. 2, 1630-1637. doi: 10.1002/adhm.201200454

Agostoni, V., Horcajada, P., Noiray, M., Malanga, M., Aykaç, A., Jicsinszky, L., et al. (2015). A "green" strategy to construct non-covalent, stable and bioactive coatings on porous MOF nanoparticles. Sci. Rep. 5:7925. doi: 10.1038/ srep07925

Aykac, A., Noiray, M., Malanga, M., Agostoni, V., Casas-Solvas, J. M., Fenyvesi, E., et al. (2017). A non-covalent "click chemistry" strategy to efficiently coat highly porous MOF nanoparticles with a stable polymeric shell. BBA Gen. Subj. 1861, 1606-1616. doi: 10.1016/j.bbagen.2017.01.016

Baati, T., Njim, L., Neffati, F., Kerkeni, A., Bouttemi, M., Gref, R., et al. (2013). In depth analysis of the in vivo toxicity of nanoparticles of porous iron(iii) metal-organic frameworks. Chem. Sci. 4, 1597-1607. doi: 10.1039/c3sc22116d

Barenholz, Y. (2012). Doxil ${ }^{\circledR}$ - The first FDA-approved nano-drug: lessons learned. J. Control. Release 160, 117-134. doi: 10.1016/j.jconrel.2012.03.020

Bellido, E., Hidalgo, T., Lozano, M. V., Guillevic, M., Simón-Vázquez, R., Santander-Ortega, M. J., et al. (2015). Heparin-engineered mesoporous iron Metal-Organic Framework nanoparticles: toward stealth drug nanocarriers. Adv. Healthc. Mater. 4, 1246-1257. doi: 10.1002/adhm.201400755

Bouffard, D. Y., Laliberté, J., and Momparler, R. L. (1993). Kinetic studies on 2'2'difluorodeoxycytidine (gemcitabine) with purified human deoxycytidine kinase and cytidine deaminase. Biochem. Pharmacol. 45, 1857-1861. doi: 10.1016/ 0006-2952(93)90444-2

Bugnicourt, L., Peers, S., Dalverny, C., and Ladavière, C. (2019). Tunable morphology of lipid/chitosan particle assemblies. J. Colloid Interface Sci. 534, 105-109. doi: 10.1016/j.jcis.2018.08.098

Chibowski, E., and Szcześ, A. (2016). Zeta potential and surface charge of DPPC and DOPC liposomes in the presence of PLC enzyme. Adsorption 22, 755-765. doi: 10.1007/s10450-016-9767-z

Cutrone, G., Li, X., Casas-Solvas, J. M., Menendez-Miranda, M., Qiu, J., Benkovics, G., et al. (2019a). Design of engineered cyclodextrin derivatives for spontaneous coating of highly porous Metal-Organic Framework nanoparticles in aqueous media. Nanomaterials 9, 1-26. doi: 10.3390/nano9081103

Cutrone, G., Qiu, J., Menendez-Miranda, M., Casas-Solvas, J. M., Aykaç, A., Li, X., et al. (2019b). Comb-like dextran copolymers: a versatile strategy to coat highly porous MOF nanoparticles with a PEG shell. Carbohydr. Polym. 223:115085. doi: 10.1016/j.carbpol.2019.115085

Giménez-marqués, M., Bellido, E., Berthelot, T., Simón-yarza, T., Hidalgo, T., Simón-vázquez, R., et al. (2018). GraftFast surface engineering to improve MOF nanoparticles furtiveness. Small 14, 1-11. doi: 10.1002/smll.201801900 and Euronanomed III (project PCInano). This work was also supported by a public grant overseen by the French National Research Agency as part of the "Investissements d'Avenir" program (Labex NanoSaclay, ANR-10-LABX-0035).

\section{ACKNOWLEDGMENTS}

We acknowledge Dr. B. Moreira-Alvarez and Dr. J. R. Encinar for their kind help with ICP-MS analysis. We also thank Dr. D. Constantin for help with XRPD experiments.

\section{SUPPLEMENTARY MATERIAL}

The Supplementary Material for this article can be found online at: https://www.frontiersin.org/articles/10.3389/fbioe. 2020.01027/full\#supplementary-material

Gref, R., Domb, A., Quellec, P., Blunk, T., Müller, R. H., Verbavatz, J. M., et al. (1995). The controlled intravenous delivery of drugs using PEG-coated sterically stabilized nanospheres. Adv. Drug Deliv. Rev. 16, 215-233. doi: 10. 1016/0169-409x(95)00026-4

Gref, R., Lück, M., Quellec, P., Marchand, M., Dellacherie, E., Harnisch, S., et al. (2000). "Stealth" corona-core nanoparticles surface modified by polyethylene glycol (PEG): Influences of the corona (PEG chain length and surface density) and of the core composition on phagocytic uptake and plasma protein adsorption. Colloids Surfaces B Biointerfaces 18, 301-313. doi: 10.1016/s09277765(99)00156-3

Gref, R., Minamitake, Y., Peracchia, M. T., Trubetskoy, V., Torchilin, V., and Langer, R. (1994). Biodegradable long-circulating polymeric nanospheres. Science 263, 1600-1603. doi: 10.1126/science.8128245

He, C., Liu, D., and Lin, W. (2015). Nanomedicine applications of hybrid nanomaterials built from Metal-Ligand Coordination bonds: Nanoscale MetalOrganic Frameworks and Nanoscale Coordination Polymers. Chem. Rev. 115, 11079-11108. doi: 10.1021/acs.chemrev.5b00125

Hidalgo, T., Bellido, E., Avila, J., Asensio, M. C., and Salles, F. (2017). Chitosancoated mesoporous MIL-100 (Fe) nanoparticles as improved bio-compatible oral nanocarriers. Sci. Rep. 100, 1-14. doi: 10.1038/srep43099

Horcajada, P., Chalati, T., Serre, C., Gillet, B., Sebrie, C., Baati, T., et al. (2010). Porous Metal-Organic-Framework nanoscale carriers as a potential platform for drug delivery and imaging. Nat. Mater. 9, 172-178. doi: 10.1038/nmat2608

Horcajada, P., Gref, R., Baati, T., Allan, P. K., Maurin, G., and Couvreur, P. (2012). Metal-Organic Frameworks in biomedicine. Chem. Rev. 112, 1232-1268. doi: $10.1021 / \mathrm{cr} 200256 \mathrm{v}$

Li, X., Lachmanski, L., Safi, S., Sene, S., Serre, C., Grenèche, J. M., et al. (2017). New insights into the degradation mechanism of Metal-Organic Frameworks drug carriers. Sci. Rep. 7, 1-17. doi: 10.1038/s41598-017-13323-1

Li, X., Porcel, E., Menendez-Miranda, M., Qiu, J., Yang, X., Pastor, A., et al. (2019a). Highly porous hybrid metal-organic nanoparticles loaded with gemcitabinemonophosphate: a multimodal approach to improve chemo and radiotherapy. ChemMedChem 15, 274-283. doi: 10.1002/cmdc.201900596

Li, X., Semiramoth, N., Hall, S., Tafani, V., Josse, J., Laurent, F., et al. (2019b). Compartmentalized encapsulation of two antibiotics in porous nanoparticles: an efficient strategy to treat intracellular infections. Part. Part. Syst. Charact. 36, 1-9. doi: 10.1002/ppsc.201970009

Luchini, A., and Vitiello, G. (2019). Understanding the nano-bio interfaces: lipidcoatings for inorganic nanoparticles as promising strategy for biomedical applications. Front. Chem. 7:343. doi: 10.3389/fchem.2019.00343

Rodriguez-Ruiz, V., Maksimenko, A., Anand, R., Monti, S., Agostoni, V., Couvreur, P., et al. (2015). Efficient "green" encapsulation of a highly hydrophilic anticancer drug in Metal-Organic Framework nanoparticles. J. Drug Target. 23, 759-767. doi: 10.3109/1061186x.2015.1073294 
Rojas, S., Arenas-Vivo, A., and Horcajada, P. (2019). Metal-Organic Frameworks: a novel platform for combined advanced therapies. Coord. Chem. Rev. 388, 202-226. doi: 10.1016/j.ccr.2019.02.032

Rosenblum, D., Joshi, N., Tao, W., Karp, J. M., and Peer, D. (2018). Progress and challenges towards targeted delivery of cancer therapeutics. Nat. Commun. 9, 1-12. doi: 10.1038/s41467-018-03705-y

Senapati, S., Mahanta, A. K., Kumar, S., and Maiti, P. (2018). Controlled drug delivery vehicles for cancer treatment and their performance. Signal Transduct. Target. Ther. 3, 1-19. doi: 10.1038/s41392-017-0004-3

Simon-Yarza, M. T., Baati, T., Paci, A., Lesueur, L. L., Seck, A., Chiper, M., et al. (2016). Antineoplastic busulfan encapsulated in Metal Organic Framework nanocarrier: first in vivo results. J. Mater. Chem. B 4, 585-588. doi: 10.1039/ c5tb02084k

Taylor-Pashow, K. M. L., Della Rocca, J., Xie, Z., Tran, S., and Lin, W. (2009). Postsynthetic modifications of iron-carboxylate nanoscale MetalOrganic Frameworks for imaging and drug delivery. J. Am. Chem. Soc. 131, 14261-14263. doi: 10.1021/ja906198y

Thevenot, J., Troutier, A., David, L., Delair, T., and Ladavière, C. (2007). Steric stabilization of lipid/polymer particle assemblies by poly (ethylene glycol)-lipids. Biomacromolecules 8, 3651-3660. doi: 10.1021/ bm700753q
Troutier, A., and Ladavière, C. (2007). An overview of lipid membrane supported by colloidal particles. Adv. Colloid Interface Sci. 133, 1-21. doi: 10.1016/j.cis. 2007.02.003

Troutier-Thuilliez, A. L., Thevenot, J., Delair, T., and Ladavière, C. (2009). Adsorption of plasmid DNA onto lipid/polymer particle assemblies. Soft. Matter. 5, 4739-4747. doi: 10.1039/b911260j

Wuttke, S., Braig, S., Preiß, T., Zimpel, A., Sicklinger, J., Bellomo, C., et al. (2015). MOF nanoparticles coated by lipid bilayers and their uptake by cancer cells. Chem. Commun. 51, 15752-15755. doi: 10.1039/c5cc06767g

Conflict of Interest: The authors declare that the research was conducted in the absence of any commercial or financial relationships that could be construed as a potential conflict of interest.

Copyright (c) 2020 Li, Salzano, Qiu, Menard, Berg, Theodossiou, Ladavière and Gref. This is an open-access article distributed under the terms of the Creative Commons Attribution License (CC BY). The use, distribution or reproduction in other forums is permitted, provided the original author(s) and the copyright owner(s) are credited and that the original publication in this journal is cited, in accordance with accepted academic practice. No use, distribution or reproduction is permitted which does not comply with these terms. 